\title{
The Effect of Sacral Neuromodulation on Anticholinergic Use and Expenditures in a Privately Insured Population
}

\author{
Jennifer T. Anger, MD, MPH ${ }^{*,+}$; Anne P. Cameron, MD; Rodger Madison ${ }^{\S}$; \\ Christopher S. Saigal, MD, MPH*; J. Quentin Clemens, MD, MSCI ; \\ The Urologic Diseases in America Project
}

\begin{abstract}
Objectives: Sacral neuromodulation is Food and Drug Administration approved for many types of voiding dysfunction. Goals of treatment often include cessation of anticholinergic therapy. With the goal of understanding the impact of sacral neuromodulation on anticholinergic use, we analyzed patterns of care using a national claims-based dataset.

Materials and Methods: The Ingenix (i3) data base contains insurance claims, including utilization and cost data, for 75 large employers. De-identified patients who underwent sacral neuromodulation between 2002 and 2007 were identified by the unique current procedural terminology-4 procedure code for pulse generator implantation, code 64590 . The number and costs of anticholinergic prescriptions were compared before and after treatment.

Results: There were 266 percutaneous and 794 two-staged procedures performed from 2002 to 2007 in the i3 dataset. A total of 484 pulse generator implantations were performed, representing $46 \%$ of the test procedures. During the year prior to pulse generator placement, each patient purchased an average of 2.1 prescriptions for an anticholinergic agent (SD 3.5). During the year after neuromodulation, each patient purchased an average of 1.0 prescription (SD 2.3, $p<0.0001$ by $t$-test). Prescription charges were $\$ 241.31$ per patient before and $\$ 103.52$ after neuromodulation, a statistically significant cost difference $(p<0.0001$ by $t$-test). During the year before the procedure, $50 \%$ of patients filled anticholinergic prescriptions. This decreased to $23 \%$ after the procedure ( $p<0.0001$ by chi-square test).

Conclusions Sacral neuromodulation was associated with a significant decrease in the use of anticholinergic medication. Costeffectiveness analyses that take into account patient quality-adjusted life years are needed to determine the true cost-benefit ratio of sacral neuromodulation.
\end{abstract}

Keywords: Claims data, cost, Ingenix (i3), overactive bladder

Conflict of Interest: All authors have no conflict of interest.

\section{INTRODUCTION}

Sacral neuromodulation is Food and Drug Administration approved for many types of voiding dysfunction refractory to medical therapy. A systematic review by Siddiqui et al. demonstrated good efficacy in the treatment of refractory overactive bladder (OAB), with acceptable surgical revision rates (1). In the case of $O A B$ symptoms, goals of treatment include the improvement in urinary frequency, urgency, and urge incontinence. For some patients, particularly those who do not tolerate anticholinergic agents, a goal of sacral neuromodulation is cessation or reduction of anticholinergic use. In some instances, anticholinergic medication is still required after sacral neuromodulation, with the combination yielding better results than one treatment alone. However, the extent to which successful neuromodulation results in a cessation is unknown or whether there is a decrease in use of anticholinergic medication.

Sacral neuromodulation is a costly therapy initially, with the implantation of the lead and pulse generator totaling $\$ 15,000$ to $\$ 22,000(2,3)$. However, the high initial cost of sacral neuromodula- tion may be offset by long-term efficacy and cost savings over time. A decrease or cessation of the use of anticholinergic medication after a successful Interstim (Medtronic, Minneapolis, MN, USA) implantation may contribute to such a cost savings. With the goal of understanding the financial and clinical impact of sacral neuromodulation on anticholinergic use, we analyzed patterns of care using a national claims-based dataset.

Address correspondence to: Jennifer T. Anger, MD, MPH, Division of Urology, Cedars-Sinai Medical Center, 99 N. La Cienega Boulevard, \#307 Beverly Hills, CA 90211, USA. Email: angerj@cshs.org

\footnotetext{
* Department of Urology, UCLA, Los Angeles, CA, USA;

+ Division of Urology, Cedars-Sinai Medical Center, Los Angeles, CA, USA;

₹ Department of Urology, University of Michigan, Ann Arbor, MI, USA; and

\& RAND Corporation, Santa Monica, CA, USA
}

For more information on author guidelines, an explanation of our peer review process, and conflict of interest informed consent policies, please go to http:// www.wiley.com/bw/submit.asp?ref=1094-7159\&site=1 
Table 1. Comparison of Prescription Usage and Cost Before and After Sacral Neuromodulation.

Year before neuromodulation
$2.1 \pm 3.5$
$\$ 241.31$
$50 \%$

Year after neuromodulation
$1.0 \pm 2.3$
$\$ 103.52$
$23 \%$

\section{MATERIALS AND METHODS}

The Ingenix (i3) database contains insurance claims for 75 large employers, covering over 30 million lives. Unlike many claims-based datasets, the i3 dataset has both utilization and cost data, including medication charges (4). This work was a secondary analysis of a larger project that sought to measure the success of sacral neuromodulation using two national datasets, Ingenix and Medicare (5). The i3 database was used to determine demographic, diagnosis, and cost information on de-identified patients who underwent sacral neuromodulation between the second quarter of 2002 and the first quarter of 2007. Each patient was linked by a unique patient identification number. Patients were initially identified by the unique current procedural terminology (CPT)-4 procedure code for a test stimulation in the sacral foramen either percutaneously (64561) or with an incision (64581), as previously described (5). International Classification of Diseases, Ninth Revision diagnosis codes identified the indication for the procedure(s), which included codes for frequency, urgency, nocturia, urge incontinence (OAB symptoms), neurogenic voiding dysfunction, interstitial cystitis, and incomplete bladder emptying or nonobstructive urinary retention. All other urologic diagnoses associated with a procedure that did not fit into one of the previously mentioned categories were grouped into the "other" category. Any person who had no urologic diagnosis whatsoever associated with their procedure was excluded, as these likely represented other types of neuromodulating devices.

Successful percutaneous test stimulation was defined as a percutaneous test followed by a simultaneous permanent lead and pulse generator implant. A successful two-stage test was defined as a surgical lead placement followed by a pulse generator placement at a later date. Those who proceeded to pulse generator implantation, CPT-4 code 64590 , were considered a treatment success, and were therefore included in this analysis. Statistical analysis was performed using Statistical Analysis Software (SAS Institute, Inc., Cary, NC, USA). The average number of anticholinergic prescriptions and the charges for prescriptions were calculated and compared before and after pulse generator implantation. $p$ Values of $\leq 0.05$ were considered statistically significant.

\section{RESULTS}

There were 266 percutaneous and 794 two-staged procedures performed from 2002 to 2007 in the Ingenix dataset. As previously described, a total of 484 pulse generator implantations were performed, representing $46 \%$ of the test procedures (5). Percutaneous procedures were successful in $24.1 \%$ of cases, compared with $50.9 \%$ following staged procedures $(p<0.0001)$. During the year prior to undergoing sacral neuromodulation, each patient purchased an average of 2.1 prescriptions for an anticholinergic agent (SD 3.5, Table 1). During the year after neuromodulation, each patient purchased an average of 1.0 prescription (SD 2.3, $p<0.0001$ by $t$-test). The largest drop in anticholinergic use was seen among patients with $O A B$ wet (from 7492 prescriptions to 5632), followed by $O A B$ dry (from 7151 to 5898, data not shown).

Anticholinergic prescription charges were $\$ 241.31$ per patient before and $\$ 103.52$ after neuromodulation, a statistically significant cost difference ( $p<0.0001$ by $t$-test, Table 1$)$. During the year before the procedure, $50 \%$ of patients filled anticholinergic prescriptions. This decreased to $23 \%$ after the procedure $(p<0.0001$ by chi-square test). Of the 112 patients who were prescribed an anticholinergic agent after neuromodulation, $40 \%$ had their last prescription less than 180 days after the implant, possibly indicating a gradual reduction in anticholinergic use over time.

\section{DISCUSSION}

We found that anticholinergic use decreased by over $50 \%$ after Interstim implantation. This may indicate significant efficacy of the device such that anticholinergic medication use was either decreased or stopped altogether. Given that many patients who begin anticholinergics are given samples (and do not necessarily fill prescriptions), we suspect that anticholinergic use in the year before sacral neuromodulation was actually higher than $50 \%$. Should this be the case, the reduction in anticholinergic use may actually be greater than what we found. Although sacral neuromodulation is usually recommended to patients whose symptoms are refractory to medical therapy, some patients proceed to Interstim because they either cannot tolerate anticholinerigcs or prefer not to take medication on a regular basis. What cannot be determined from this dataset is the reason for cessation of anticholinergic use. It is possible that some patients stopped medication use for reasons other than success of the pulse generator, such as side-effects or lack of efficacy of medication. Also, cessation could have occurred before the actual device was implanted. In addition, this population included a small percentage of patients $(9.5 \%)$ who underwent Interstim therapy for urinary retention, and anticholinergic use would only be used in those patients with incomplete bladder emptying and concomitant irritative symptoms (vs. complete retention) (5). Regardless, our findings shed light on the relationship between sacral neuromodulation and anticholinergic use among all patients undergoing Interstim.

The decrease in use of anticholinergics in the year following pulse generator implantation resulted in a cost savings of $\$ 137.79$ in prescription charges. This is consistent with the findings of Aboseif et al., who found that drug expenditures decreased by $30 \%$ after Interstim in 65 patients, from $\$ 693$ to $\$ 483$ per patient $(p<0.02)$. They also found a mean decrease in office visits of 2.2 visits in the year after implant, which corresponds to a $73 \%$ reduction in office visit expenses, from $\$ 994$ to $\$ 265$ per patient (6). From a financial perspective, this savings in prescription charges appears quite small compared with the initial cost of the Interstim pulse generator. Though sacral neuromodulation is costly initially, and has been shown to be more costly than repeated injections of botulinum toxin after two years (7), sacral neuromodulation becomes more 
cost-effective than other modalities after five years (8). In addition, long-term cessation of anticholinergic medication, with its associated side-effects of dry mouth, dry eyes, and constipation, can significantly improve one's quality of life, providing more than a simple financial savings. Though patients in the i3 database continued to fill an average of one prescription in the year after Interstim, a large minority of these occurred in the first six months after the implant, suggesting that medication use was stopped over time. What cannot be measured in claims data is the impact on health-related quality of life that these patients experienced as a result of Interstim.

\section{CONCLUSIONS}

Sacral neuromodulation was associated with a significant decrease in use of anticholinergic medication, as evidenced by fewer expenditures on anticholinergic agents and 50\% fewer prescriptions filled. In order to determine the true cost-benefit ratio of sacral neuromodulation, cost-effectiveness analyses that take into account patient quality-adjusted life years and costs over time are needed.

\section{Acknowledgement}

Funding for this study was provided by the National Institute of Diabetes and Digestive and Kidney Diseases as part of the Urologic Diseases in America Project.

\section{Authorship Statements}

Drs. Anger, Cameron, Saigal, and Clemens and Mr. Madison designed and conducted the study, including data analysis. Dr. Anger prepared the initial manuscript draft with important intellectual input from Drs. Cameron, Saigal, and Clemens, and Mr. Madison. All authors worked on and critically reviewed subsequent drafts. All authors approved the final manuscript.

\section{How to Cite this Article:}

Anger J.T., Cameron A.P., Madison R., Saigal C.S., Clemens J.Q., The Urologic Diseases in America Project 2014. The Effect of Sacral Neuromodulation on Anticholinergic Use and Expenditures in a Privately Insured Population.

Neuromodulation 2014; 17: 72-74

\section{REFERENCES}

1. Siddiqui NY, Wu JM, Amundsen CL. Efficacy and adverse events of sacral nerve stimulation for overactive bladder: a systematic review. Neurourol Urodyn 2010;29 (Suppl 1):S18-S23.

2. Siddiqui NY, Amundsen CL, Visco AG, Myers ER, Wu JM. Cost-effectiveness of sacral neuromodulation versus intravesical botulinum $A$ toxin for treatment of refractory urge incontinence. J Urol 2009;182:2799-2804.

3. Watanabe JH, Campbell JD, Ravelo A, Chancellor MB, Kowalski J, Sullivan SD. Cost analysis of interventions for antimuscarinic refractory patients with overactive bladder. Urology 2010;76:835-840.

4. Saigal CS, Joyce GF, Geschwind SA, Litwin MS. Methods. In: Litwin MS, Saigal CS, eds. Urologic diseases in America. US Department of Health and Human Services, Public Health Service, National Institutes of Health, National Institute of Diabetes and Digestive and Kidney Diseases. Washington, DC: US Government Publishing Office, 2004; NIH Publication No. 04-5512, pp. 283-316.

5. Cameron AP, Anger JT, Madison R, Saigal CS, Clemens JQ. National trends in the usage and success of sacral nerve stimulation. J Urol 2011;185:970-975.

6. Aboseif SR, Kim DH, Rieder JM et al. Sacral neuromodulation: cost considerations and clinical benefits. Urology 2007;70:1069-1073.

7. Siddiqui NY, Amundsen CL, Visco AG, Myers ER, Wu JM. Cost-effectiveness of sacral neuromodulation versus intravesical botulinum A toxin for treatment of refractory urge incontinence. J Urol 2009;182:2944-2950.

8. Leong RK, de Wachter SGG, Joore MA, van Kerrebroeck PEV. Cost-effectiveness analysis of sacral neuromodulation and botulinum toxin A treatment for patients with idiopathic overactive bladder. BJU Int 2011;108:558-564.

\section{COMMENTS}

This is a retrospective study analyzing usage of anticholinergics after neuromodulation implant. The study was done using private insurance database. It is a well designed and needed study. The results indicate significant reduction in usage of anticholinergic medication. I have no specific comments regarding the paper since it is written very well.

Jerzy Gajewski, M.D. Halifax, Nova Scotia, Canada

$$
* * *
$$

Sacral Neuromodulation (SNM) is an FDA approved minimally invasive therapy for voiding dysfunction. Its efficacy on improving patient LUTS and quality of life is documented in the literature. This study reports anticholinergic use and expenditure in a privately insured population.

I found it a good piece of information to share in that there was a significant drop of anticholinergic prescription charges post implantation (from \$241.31 per patient to \$103.52 after neuromodulation implant). This is important information to share especially in countries where the SNM is not covered by health agencies and insurance companies.

I believe that in the near future with the available cost effectiveness published data and expanding indications, SNM will be used more often.

Dr. Mai Banakhar Jeddah, Saudi Arabia

Comments not included in the Early View version of this paper. 